\title{
COMPOSICIÓN FLORÍSTICA DE UN BOSQUE MESÓFILO Del CENTRO de Veracruz, MÉXICO
}

\author{
José G. García Franco ${ }^{1,3}$, Gonzalo Castillo-Campos², Klaus Mehltreter ${ }^{1}$, \\ María Luisa Martínez ${ }^{1}$ y Gabriela VázQueZ ${ }^{1}$ \\ 'Departamento Ecología Funcional \\ ${ }^{2}$ Departamento Biodiversidad y Sistemática \\ Instituto de Ecología, A.C. Km 2.5 Carretera Antigua a Coatepec, CP 91070 Xalapa Veracruz, México \\ ${ }^{3}$ Autor para la correspondencia. Correo-e: franco@inecol.edu.mx
}

\begin{abstract}
Resumen: En la región centro de Veracruz existen fragmentos de bosque mesófilo de montaña (BMM) que no se han explorado. Nosotros estudiamos la composición florística y algunos aspectos de la estructura de un BMM en la localidad de La Cortadura. El muestreo de leñosas, herbáceas y epífitas vasculares se realizó en tres microcuencas (1000 $\mathrm{m}^{2}$ cada una). Las áreas muestreadas tuvieron gran cobertura con árboles de buena altura, algunos emergentes, y la proporción de formas de vida fue igual entre microcuencas. Se registraron 258 especies ( 150 géneros, 82 familias), pero el bosque en su interior es heterogéneo. Aunque el número de especies en cada microcuenca fue parecido (144-163), las tres microcuencas sólo compartieron 55 especies (34-38\%). El BMM de La Cortadura está muy bien conservado, tiene 58 y $31 \%$ de las familias que habitan preferentemente o exclusivas en este tipo de vegetación, y se han encontrado nuevos registros de distribución y una nueva especie.
\end{abstract}

Palabras Clave: riqueza de especies, fragmentación, diversidad, heterogeneidad, La Cortadura.

\begin{abstract}
In the central area of Veracruz a few remnants of cloud forest (CF) have not been explored previously. We studied the floristic composition and some structural features of one of these remnants of CF in La Cortadura. Sampling of woody plants, herbs and vascular epiphytic species was made in three river basins (plots of $1000 \mathrm{~m}^{2}$ each). The sampled areas showed dense canopy cover, some emergent tree species, and similar life form proportions. We recorded 258 species ( 150 genera, 82 families), however the forest is heterogeneous. Even though the number of species found in each river basin was nearly similar (144-163), they only shared 55 species (34-38\%). The CF at La Cortadura is well preserved and contains 58 and $31 \%$ of the preferred or exclusive families for this vegetation type. In addition, some new distribution records and a new species were recorded for the area.
\end{abstract}

Key Words: species richness, fragmentation, diversity, heterogeneity, La Cortadura.

E bosque mesófilo de montaña (BMM) (sensu Rzedowski, 1978), es un tipo de vegetación complejo y heterogéneo que comprende diferentes tipos de bosques, los cuales responden en su composición y fisonomía a las diferentes condiciones geográficas y ecológicas en las que se distribuyen (Luna et al., 2001). El BMM es el más diverso por unidad de área en México y, aunque no llega a cubrir más del 1\% del territorio nacional (Rzedowski, 1996), contribuye con cerca del $10 \%$ a la diversidad vegetal de México, con un alto porcentaje de endemismo (Rzedowski, 1993). Su distribución es discontinua en los sistemas montañosos de México, y obedece a condiciones particulares de altitud, humedad y temperatura, que se presentan en las laderas de la Sierra Madre Occidental (Durango, Jalisco, Nayarit, Sinaloa, So- nora), Sierra Madre Oriental (Hidalgo, Nuevo León, Oaxaca, Puebla, Querétaro, San Luís Potosí, Tamaulipas y Veracruz), así como en ciertas zonas de Chiapas, Guerrero, Jalisco y Michoacán. Además de la importancia que el BMM tiene como ecosistema con gran biodiversidad, por su ubicación en las montañas, principalmente en sitios con orografía abrupta, recientemente se le ha reconocido su gran contribución en proporcionar servicios ambientales, como la captura de carbono, la disminución de la erosión, de deslaves y de inundaciones, y el mantenimiento de las corrientes de agua (Cavelier y Vargas, 2002; Manson, 2004).

En el centro de Veracruz el BMM está representado por remanentes inmersos en un paisaje compuesto por una matriz de campos agrícolas, potreros, cafetales y asentamien-

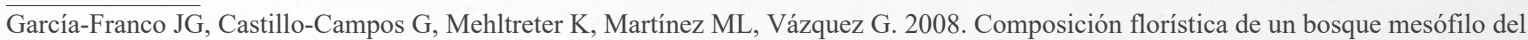
centro de Veracruz, México. Boletín de la Sociedad Botánica de México 83: 37-52. 
tos humanos (Williams-Linera, 1993). La franja de BMM se encuentra entre 1200-2100 m.s.n.m. en lomeríos con suelos volcánicos con una gran heterogeneidad topográfica y ambiental. Estos bosques se desarrollan comúnmente en un clima templado húmedo con lluvias todo el año (WilliamsLinera, 2007). La gran variación topográfica y micro ambiental ha favorecido que se presenten considerables diferencias en la composición y estructura entre fragmentos de BMM relativamente cercanos. Diversos estudios florísticos y ecológicos se han realizado para conocer la riqueza particular de los fragmentos y entender su funcionamiento como ecosistema (Williams-Linera, 1997, 2002; Mehltreter et al., 2005; Williams-Linera et al., 2005; Flores-Palacios y García-Franco, 2006; Heredia et al., 2006; Mehltreter y GarcíaFranco, 2008; entre otros). A pesar de la riqueza biológica y de los servicios ambientales que proporciona, la tasa de conversión del BMM a diferentes usos de suelo antropogénicos (agroforestales, pecuarios y urbanos) es muy alta. Por ejemplo, en los alrededores de la ciudad de Xalapa sólo queda $10 \%$ de la cobertura original, por el crecimiento desordenado de las actividades antropógenas (Williams-Linera, 2007). Sin embargo, existen algunos remanentes que por su lejanía a los centros urbanos y por su orografía relativamente inaccesible no han sido explorados y aún se encuentran en excelentes condiciones de conservación.

El objetivo del trabajo es contribuir al conocimiento florístico de los bosques mesófilos de montaña, en particular de aquellos que se distribuyen en el centro del estado de Veracruz. En este sentido, se analizó la vegetación del BMM de La Cortadura que constituye uno de los pocos remanentes bien conservados, a pesar de encontrarse en un paisaje antropizado.

\section{Materiales y métodos}

El estudio se realizó en la localidad de La Cortadura, ubicada en la zona montañosa central del estado de Veracruz, México (figura 1). La región de La Cortadura está cubierta por BMM y se localiza en la ladera oriente del Cofre de Perote entre 1,800 y 2,000 m.s.n.m., donde predomina el suelo de tipo andosol húmico de origen volcánico. El sitio está conformado por una reserva municipal (Municipio de Coatepec) y propiedades privadas. Esta parte del estado de Veracruz se encuentra relativamente bien conservada ya

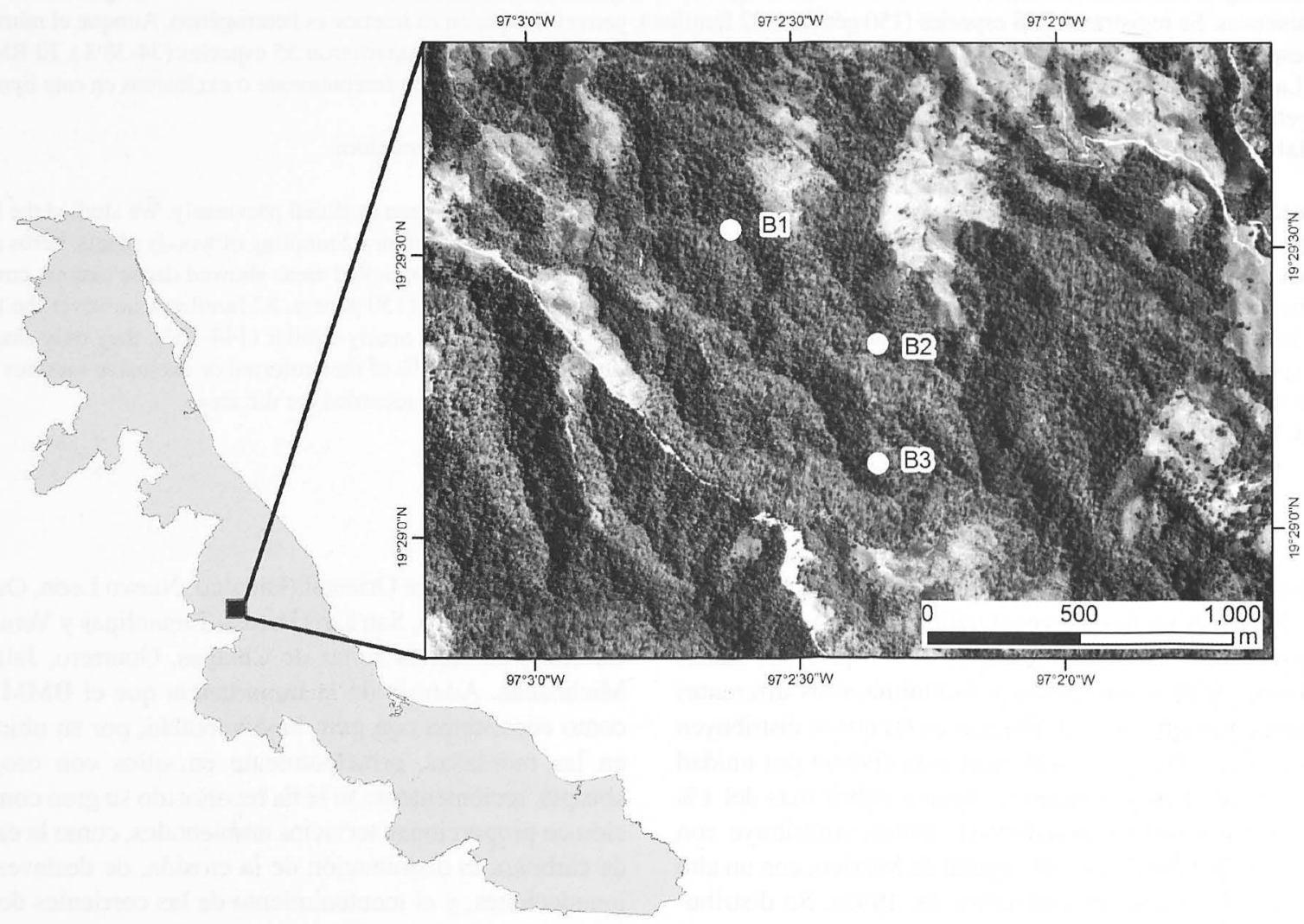

Figura 1. Localización de La Cortadura y de las microcuencas estudiadas (B1, B2 y B3), en la zona montañosa del centro de Veracruz, México. (Fuente del mapa. Contorno del estado de Veracruz: Comisión Nacional para el Conocimiento y Uso de la Biodiversidad (2002). Política Estatal. Escala 1:1000 000. Imagen: Ortofoto digital E14B36C de fotografías aéreas escala 1:75,000 de Mayo de 1995, INEGI). 
que el $80 \%$ de la superficie corresponde a BMM, y el $20 \%$ a otros usos de suelo (figura 1). El clima de esta zona es templado húmedo con una temperatura promedio de $18^{\circ} \mathrm{C}$ (mínima $10-14^{\circ} \mathrm{C}$ y máxima $20-23^{\circ} \mathrm{C}$ ), y una precipitación anual de 1,500 mm distribuida a lo largo del año en las temporadas de nortes (noviembre-marzo, fría; y húmeda) y de lluvias (julio-septiembre, cálida; con abundante precipitación) (Williams-Linera, 2007).

El terreno en la zona es sumamente irregular, con pendientes muy abruptas, y en el fondo de las cañadas se inician los escurrimientos de agua que conforman la parte alta de la Cuenca del Río La Antigua. Esta cuenca del estado de Veracruz $\left(2,326 \mathrm{~km}^{2}, 0-3,000 \mathrm{~m}\right.$ s.n.m.) está considerada por la CONABIO (2000a, 2000b) como área de alta biodiversidad (AAB), Área Prioritaria Terrestre (Pico de Orizaba-Cofre de Perote) y región Hidrológica Prioritaria (Golfo de México: Río La Antigua) (Muñoz-Villers y López-Blanco, 2008; Pérez-Maqueo et al., 2007).

Se seleccionaron tres microcuencas (en adelante B1, B2 y B3; ver figura 1) que cumplieron los siguientes requisitos: que fueran relativamente accesibles, que sus laderas tuvieran al menos $90 \mathrm{~m}$ antes de llegar a la cresta, que tuvieran ríos de primer orden y una longitud de al menos $150 \mathrm{~m}$ antes de cambiar a ríos de segundo orden, y que no mostraran rasgos recientes de perturbación antropógena (e.g. caminos, tocones o pastoreo). Con la idea de incluir en el muestreo la posible variación en la vegetación por la distancia al agua y la orientación de las pendientes, en cada microcuenca se muestrearon $1,000 \mathrm{~m}^{2}$ por medio de dos transectos perpendiculares a la corriente y dos paralelos a ella. En los transectos perpendiculares se trazaron tres cuadros de muestreo cada $25 \mathrm{~m}$ de distancia (a 25, 50 y $75 \mathrm{~m}$ de la parte baja de la cañada a la cresta). A lo largo de cada uno de los transectos paralelos, ubicados entre 5-10 m de la parte baja de la microcuenca, se hicieron dos cuadros de muestreo separados por $25 \mathrm{~m}$ (25 y $50 \mathrm{~m})$. En cada cuadro $(10 \times 10 \mathrm{~m})$ se hizo una evaluación ambiental general, considerando la cobertura de leñosas, cobertura de herbáceas, suelo desnudo, pedregosidad (todos los anteriores como porcentaje relacionados al cuadro de muestreo), altura y DAP de todos los árboles enraizados encontrados en cada cuadro, y la altura máxima de cada especie arbustiva y herbácea. El registro de las especies de hierbas se realizó en 3 cuadros de 2 × 2 m ubicados al azar dentro del cuadro de $10 \times 10 \mathrm{~m}$. Las especies de epífitas vasculares se registraron por medio de binoculares en los árboles presentes dentro de los cuadros de $10 \times 10 \mathrm{~m}$. Los ejemplares de respaldo se depositaron en el herbario (XAL) del Instituto de Ecología, A.C.

Se construyó una lista de todas las especies identificadas, siguiendo la clasificación de Cronquist (1988). Cuando esto no fue posible se siguió la clasificación y el registro de especies en la Flora de Veracruz (Sosa y Gómez-Pompa, 1994), así como la nomenclatura empleada en los fascículos de la Flora de Veracruz (v.gr., Espejo-Serna et al., 2005).
Mientras que para los helechos y afines se siguió la nomenclatura que Mickel y Smith (2004) utilizaron en su obra de las Pteridophyta de México. Los valores promedio de los parámetros ambientales registrados en cada microcuenca se compararon por medio de análisis de varianza (SigmaStat 3.1). Cuando fue necesario, los datos se transformaron mediante la raíz cuadrada del arcoseno de $i$ para el porcentaje, y logaritmo de $i$ en datos continuos antes del análisis (Zar, 1996). La proporción de formas de vida de cada microcuenca fue comparada mediante una tabla de contingencia (SigmaStat 3.1). Para cada microcuenca se determinó el número de especies por formas de vida, la riqueza de especies (S), y los índices de similitud de Jaccard (Magurran, 2004). Se aplicó un Análisis de Componentes Principales para detectar algún gradiente espacial entre las microcuencas, considerando en este análisis la presencia - ausencia de las especies y las características ambientales registradas (MVSP V 3.13).

\section{Resultados}

Características ambientales y estructura. Las microcuencas estudiadas presentan una fuerte pendiente $(38 \pm 9.5$ grados, $0 \pm$ D.E.), con un bajo porcentaje de suelo desnudo y pedregosidad $(5.3 \pm 14.9$ y $8.7 \pm 13.2$, respectivamente $)$, y un elevado porcentaje de cobertura tanto de leñosas ( 91.3 $\pm 10.7)$ como de herbáceas $(70.3 \pm 19.2)$. La cobertura de herbáceas fue variable entre las microcuencas $(F=3.88$, P $=0.03$ ), siendo significativamente mayor en la microcuenca B1 ( $82 \pm 12.3$; comparación múltiple de Holm-Sidak). La altura promedio de los árboles en el sitio es de $27.1 \mathrm{~m} \mathrm{(} \pm$ $9.9)$ con un DAP promedio de $28.68 \mathrm{~cm}( \pm 22.38)$. No se detectaron diferencias significativas para el DAP entre las tres microcuencas $(F=1.82, \mathrm{P}=0.181)$. Sin embargo, dispersos y poco frecuentes se presentan algunos árboles emergentes como Meliosma alba y Quercus spp. que alcanzan alturas de más de $40 \mathrm{~m}$ y hasta $156 \mathrm{~cm}$ de DAP. El estrato arbustivo tiene en promedio una altura de $4.1 \mathrm{~m}( \pm 1.6)$, el cual no fue significativamente diferente entre microcuencas $(F=1.324$, $\mathrm{P}=0.283$ ).

Los árboles y las hierbas fueron las formas de vida con mayor número de especies (figura 2). En las tres microcuencas se encontraron 119 especies leñosas y 139 herbáceas. La proporción de especies en cada forma de vida fue semejante en cada una de las microcuencas $\left(\chi^{2}=1.27, P=0.973\right.$; árboles $26-28 \%$, arbustos $11-15 \%$, bejucos $4-6 \%$ y hierbas $54-56 \%)$.

No todas las especies registradas por forma de vida estuvieron presentes en las tres microcuencas estudiadas. De las 67 especies de árboles registradas, sólo 15 estuvieron presentes en las tres microcuencas (v.gr., Parathesis melanosticta, Hedyosmum mexicanum, Miconia chysoneura, Alchornea latifolia, Miconia glaberrima, Cyathea fulva, Turpinia occidentalis y Clethra mexicana; de la más a la menos frecuente); mientras que de las 35 especies de arbustos, sólo 


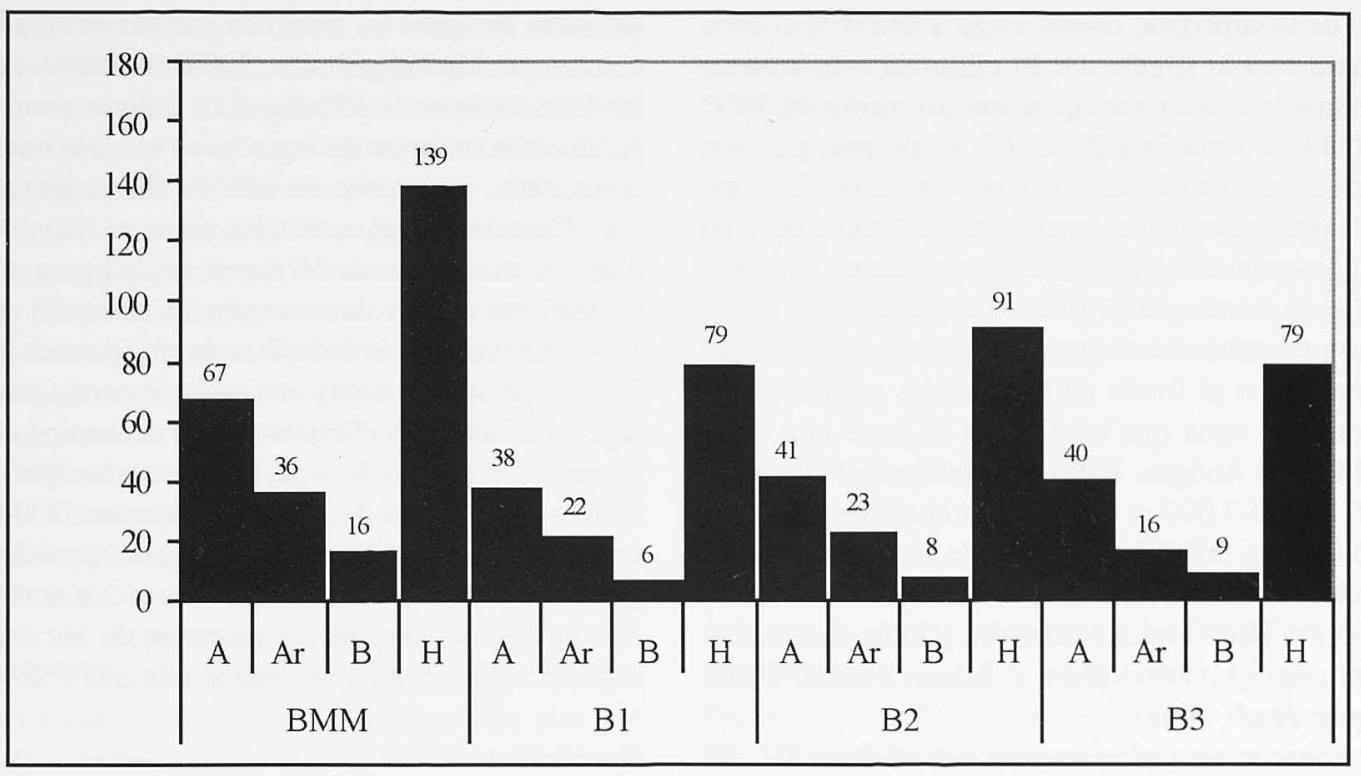

Figura 2. Formas de vida registradas en el BMM de La Cortadura y en cada una de las microcuencas estudiadas $(\mathrm{B} 1=$ microcuenca 1 , B2 $=$ microcuenca $2, \mathrm{~B} 3=$ microcuenca $3, \mathrm{~A}=$ árbol, $\mathrm{Ar}=$ arbusto, $\mathrm{B}=$ bejuco, $\mathrm{H}=$ hierba). Se indica sobre las barras el número de especies registrado.

siete se encontraron en las tres microcuencas (v.gr., Hoffmannia excelsa, Psychotria sp., Lycianthes geminiflora, Moussonia deppeana y Deppea grandiflora; de la más a la menos frecuente); sólo dos especies de bejucos se registraron en las tres microcuencas (v.gr., Smilax jalapensis y Smilax mollis), y de las 139 especies de hierbas 31 aparecieron en las tres microcuencas (v.gr., Polypodium falcaria, Arachniodes denticulada, Roldana lanicaulis, Nopalxochia phyllanthoides, Peperomia aff. quadrifolia, Polypodium longepinnulatum y Anthurium scandens; de la más a la menos frecuente). Por otro lado, encontramos especies exclusivas de las diferentes microcuencas. En una sola microcuenca se registraron 30 especies de árboles (v.gr, en B1 Dicksonia sellowiana, Miconia oligotricha, Psychotria galleotiana; en B2 Sambucus nigra subsp. canadensis, Sideroxylon capiri, Symplocos coccinea,; en B3 Myrsine coriacea, Ocotea psychotrioides, Vaccinium leucanthum), 18 especies de arbustos (v.gr., en B1 Fuchsia microphylla, Solanum nudum, S. pubigerum; en B2 S. rovirosanum, Urera caracasana, Witheringia solanacea; en B3 Bartlettina xalapanan, Cestrum elegans, Prunus samydoides), 11 especies de bejucos (v.gr., en B1 Cyclanthera langaei; en B2 Hanburia mexicana, Serjania sp., Vitis bourgaeana; en B3 Marcgravia stonei, Macleania sp., Passiflora sexflora) y 60 especies de hierbas (v.gr., en B1 Blechnum stoloniferum, Elaphoglossum peltatum, Rhynchospora aristata; en B2 Ichnanthus pallens, Megalastrum pulverulentum, Polypodium sp. 1; en B3 Peperomia tetrophylla, Sticherus palmatus, Tillandsia filifolia). En el Apéndice 1 se detallan las especies más frecuentes y el número de cuadros en donde se registraron.

Composición florística. Se registró un total de 258 especies vasculares pertenecientes a 150 géneros y 82 familias. De las familias encontradas, 19 fueron pteridofitas, 10 monocotiledóneas y 53 dicotiledóneas. Las familias con más especies fueron, Polypodiaceae (13) y Aspleniaceae (8) en las pteridofitas; Bromeliaceae (18) y Orchidaceae (10) en las monocotiledoneas, y Piperaceae (13), Rubiaceae (11) y Solanaceae (10) de las dicotiledóneas (cuadro 1).

El 54\% (19 de 35, v.gr., Alsophila firma) de los géneros de pteridofitas tuvieron una sola especie, mientras que el $72 \%$ (21 de 29) de los géneros de monocotiledóneas presentaron una especie (v.gr., Greigia vanhyningii), y $77 \%$ (67 de 87) de los géneros de las dicotiledóneas (v.gr., Hedyosmum mexicanum) también presentaron una sola especie. Los géneros con más especies fueron Asplenium (8), Polypodium (7) y Elaphoglossum (6) de las pteridofitas; Tillandsia (12) de las monocotiledóneas; y Peperomia (8), Solanum (5) y Quercus (5) de las dicotiledóneas.

Las microcuencas de La Cortadura presentan una gran heterogeneidad entre ellas. El análisis de clasificación indica que hay una separación entre los cuadros muestreados en el bosque (figura 3). En general, los cuadros de una misma microcuenca se agrupan en el mismo grupo, aparentemente debido a las especies y características ambientales similares que presentan (ver apéndice 1).

Aunque las microcuencas B1 y B2 parecen más semejantes entre sí, el análisis de ordenación indica que los cuadros de cada una de las microcuencas se separan hacia extremos diferentes dentro del espacio de ordenación (figura 4). Aparentemente esta agregación y separación se debe a las especies únicas de cada microcuenca, a las que se comparten entre pares de microcuencas y a aquellas que se presentan 
Cuadro 1. Familias con el número de especies registradas en el BMM de La Cortadura, Veracruz.

\begin{tabular}{|c|c|}
\hline Grupo /Familia & Especies \\
\hline \multicolumn{2}{|l|}{ PTERIDOPHYTAS } \\
\hline Aspleniaceae & 8 \\
\hline Blechnaceae & 5 \\
\hline Cyatheaceae & 3 \\
\hline Dennstaedtiaceae & 4 \\
\hline Dicksoniaceae & 3 \\
\hline Dryopteridaceae & 7 \\
\hline Grammitidaceae & 4 \\
\hline Gleicheniaceae & 1 \\
\hline Hymenophyllaceae & 7 \\
\hline Lomariopsidaceae & 6 \\
\hline Lycopodiaceae & 1 \\
\hline Marattiaceae & 1 \\
\hline Polypodiaceae & 13 \\
\hline Pšilotaceae & 1 \\
\hline Pteridaceae & 3 \\
\hline Selaginellaceae & 2 \\
\hline Thelypteridaceae & 2 \\
\hline Woodsiaceae & 4 \\
\hline Vittariaceae & 2 \\
\hline \multicolumn{2}{|c|}{ MONOCOTILEDONEAS } \\
\hline Araceae & 5 \\
\hline Arecaceae & 3 \\
\hline Bromeliaceae & 18 \\
\hline Commelinaceae & 2 \\
\hline Convallariaceae & 1 \\
\hline Cyperaceae & 4 \\
\hline Orchidaceae & 10 \\
\hline Poaceae & 4 \\
\hline Podocarpaceae & 1 \\
\hline Smilacaceae & 3 \\
\hline \multicolumn{2}{|l|}{ DICOTILEDONEAS } \\
\hline Acanthaceae & 1 \\
\hline Actinidiaceae & 1 \\
\hline Amaranthaceae & 1 \\
\hline Aquifoliaceae & 1 \\
\hline Araliaceae & 6 \\
\hline Asclepiadaceae & 1 \\
\hline Asteraceae & 5 \\
\hline Cactaceae & 1 \\
\hline Campanulaceae & 1 \\
\hline Caprifoliaceae & 1 \\
\hline
\end{tabular}

\begin{tabular}{|c|c|}
\hline Grupo /Familia & Especies \\
\hline Celastraceae & 3 \\
\hline Clethraceae & 2 \\
\hline Chloranthaceae & 1 \\
\hline Cochlospermaceae & 1 \\
\hline Crassulaceae & 1 \\
\hline Cucurbitaceae & 2 \\
\hline Cunoniaceae & 1 \\
\hline Ericaceae & 4 \\
\hline Euphorbiaceae & 3 \\
\hline Fabaceae & 2 \\
\hline Fagaceae & 5 \\
\hline Gesneriaceae & 1 \\
\hline Hamamelidaceae & 1 \\
\hline Lamiaceae & 1 \\
\hline Lauraceae & 5 \\
\hline Magnoliaceae & 1 \\
\hline Marcgraviaceae & 1 \\
\hline Melastomataceae & 5 \\
\hline Meliaceae & 2 \\
\hline Myrsinaceae & 2 \\
\hline Myrtaceae & 1 \\
\hline Onagraceae & 1 \\
\hline Passifloraceae & 1 \\
\hline Phyllonomaceae & 1 \\
\hline Piperaceae & 13 \\
\hline Rhamnaceae & 2 \\
\hline Rosaceae & 4 \\
\hline Rubiaceae & 11 \\
\hline Rutaceae & 4 \\
\hline Sabiaceae & 1 \\
\hline Sapindaceae & 3 \\
\hline Simaroubaceae & 1 \\
\hline Solanaceae & 10 \\
\hline Staphyleaceae & 1 \\
\hline Styracaceae & 1 \\
\hline Symplocaceae & 1 \\
\hline Theaceae & 1 \\
\hline Thymelaeaceae & 1 \\
\hline Urticaceae & 4 \\
\hline Verbenaceae & 1 \\
\hline Viburnaceae & 1 \\
\hline Viscaceae & 1 \\
\hline Vitaceae & 1 \\
\hline
\end{tabular}

en todas ellas, ya que el número de especies registrado en cada microcuenca es muy cercano $(\mathrm{B} 1=145$ especies, $\mathrm{B} 2=$ 163 especies y B3 $=144$ especies).

La figura 5 muestra el conjunto de especies únicas y compartidas entre las microcuencas. La microcuenca B3 presentó el mayor número de especies exclusivas (33\%), mientras que $\mathrm{B} 1$ y $\mathrm{B} 2$ tuvieron números muy semejantes (27 y 23\%, respectivamente). Las tres microcuencas compartieron 55 especies que representaron de 38 a $38 \%$ del total de las especies en cada una. Por otro lado, la similitud no fue muy alta entre las microcuencas, y esta varió entre 0.30 y

0.47 (índice de similitud de Jaccard). La más baja se encontró entre las microcuencas más alejadas entre sí (B1 y B3).

\section{Discusión}

El BMM de La Cortadura es un remanente bastante bien conservado en el centro de Veracruz. La lejanía de centros urbanos y su orografía fuertemente accidentada, han impedido su transformación hacia otro uso de suelo. La estructura de la vegetación indica un sitio conservado con una densa cobertura arbórea con árboles emergentes de hasta $40 \mathrm{~m}$ de 


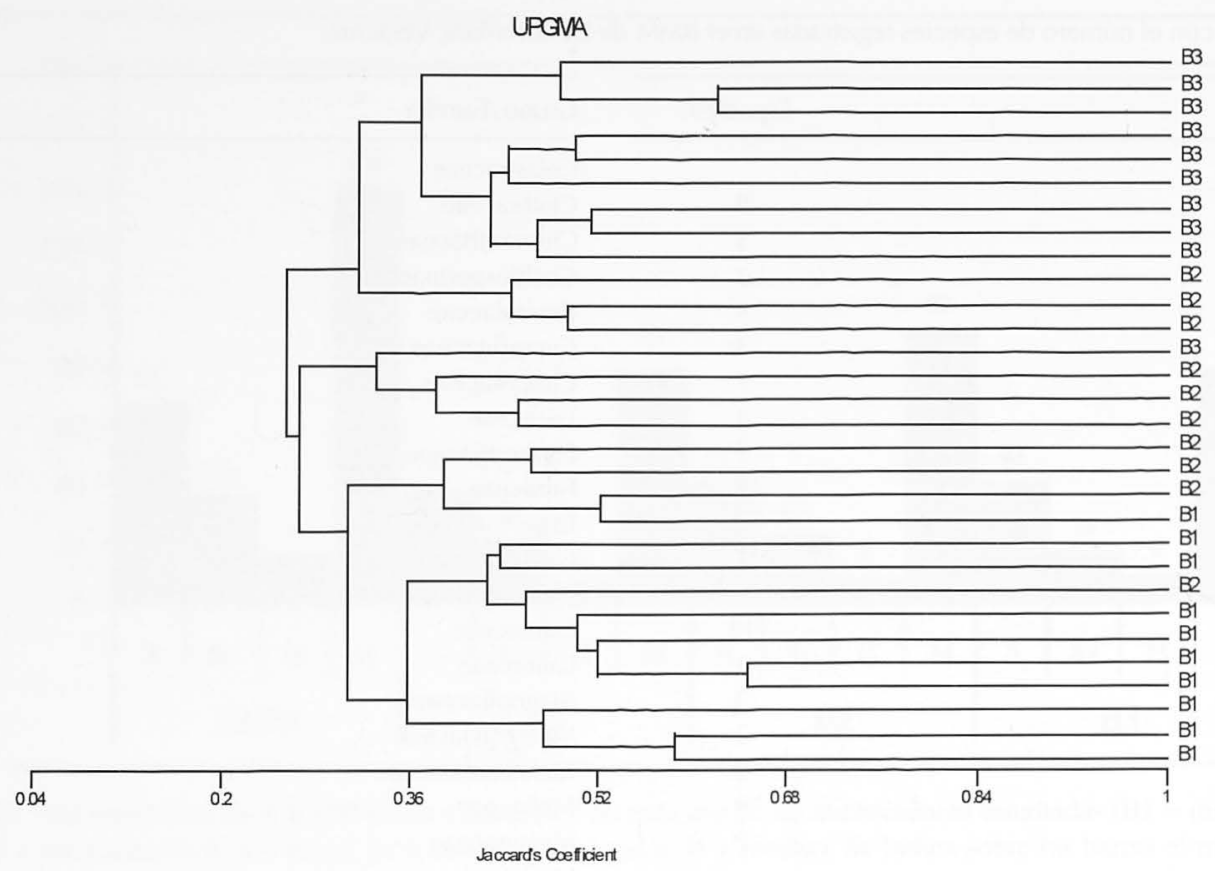

Figura 3. Dendrograma del análisis de similitud de los cuadros de muestreo. Los cuadros de la misma microcuenca (indicados como B1, B2 y B3) se agrupan en los mismos clados o en clados relacionados. Las líneas punteadas indican agrupación de cuadros, presumiblemente, con características muy semejantes.

altura y un dosel con altura promedio de $27 \mathrm{~m}$, lo cual es semejante a lo observado en otras comunidades de bosque mesófilo de montaña de Veracruz (Luna et al., 2001). A pesar de ser un sitio con fuerte pendiente y expuesto al efecto del viento (huracanes, tormentas tropicales y vientos de invierno), no existen rastros de disturbios naturales catastróficos, y eso ha permitido que algunos árboles alcancen alturas superiores a los $40 \mathrm{~m}$. El número de 258 especies registradas en el BMM de La Cortadura es alto, considerando la superficie muestreada $\left(3,000 \mathrm{~m}^{2}\right)$ y el esfuerzo de muestreo realizado (15 días en el campo). En un BMM de la región cercana de Teocelo, Veracruz. Luna et al. (1988) registraron 271 especies, mientras que en tres localidades de BMM del estado de Hidalgo se registraron entre 359 y 394 especies (Alcántara y Luna, 2001; Ponce-Vargas et al., 2006); sin embargo, en un bosque mesófilo de la Sierra Gorda de Querétaro registraron 774 especies, aunque el esfuerzo de muestreo fue mayor (14 salidas mensuales de marzo de 1998 a abril de 2000, 4-5 días cada mes) (Cartujano et al., 2002). Por otro lado, el $58 \%$ de las familias que Rzedowski (1996) considera que prosperan preferentemente en el BMM se encontraron en La Cortadura, familias como Cyatheaceae, Gesneriaceae, Hymenophyllaceae, Magnoliaceae, Orchidaceae, Selaginellaceae, Theaceae y Thymelaeaceae; y el $31 \%$ de las familias exclusivas o casi exclusivas al BMM, como Chloranthaceae, Cunoniaceae, Hamamelidaceae y Podocarpaceae. Además, el 46\% de algunos de los géneros cuantitativamente importantes del BMM (Rzedowski, 1996) también fueron registrados en el
BMM de La Cortadura, como Cinnamomum, Clethra, Dendropanax, Liquidambar, Meliosma, Oreopanax, Persea y Quercus, entre otros. Es seguro que realizando colectas en otras microcuencas de La Cortadura, el número de especies registradas pudiera incrementarse. Se estima que el muestreo de las epífitas vasculares con ascensos a los árboles debe incrementarse por lo menos en un 16-20\% el registro de especies (Flores-Palacios y García-Franco, 2001).

De acuerdo con la Norma Oficial Mexicana (SEMARNAT, 2002) se registraron 10 especies en peligro de extinción y 3 especies endémicas al BMM, algunas relativamente frecuentes con gran cobertura (Cyathea fulva y Marattia laxa), mientras que otras poco frecuentes y baja cobertura (Cyathea divergens y Psilotum complanatum). Por otro lado, las zonas de BMM donde se distribuye actualmente Podocarpus matudae son muy escasas, y esta especie se encuentra con una abundancia de escasa a regular en La Cortadura. También en La Cortadura se registraron dos especies, Ponthieva brenesii (Orchidaceae) y Piper xanthostachyum (Piperaceae), que son nuevos registros de distribución para el estado de Veracruz (Castillo-Campos et al., en preparación); al igual que se tiene a la fecha una nueva especie de Psychotria (Castillo-Campos et al., en preparación). Lo anterior resalta la importancia del sitio por estar bien conservado, ser refugio de biodiversidad, y representar un reducto importante de BMM en el centro de Veracruz. La heterogeneidad del BMM a lo largo de su distribución, como característica innata, ha sido señalada 


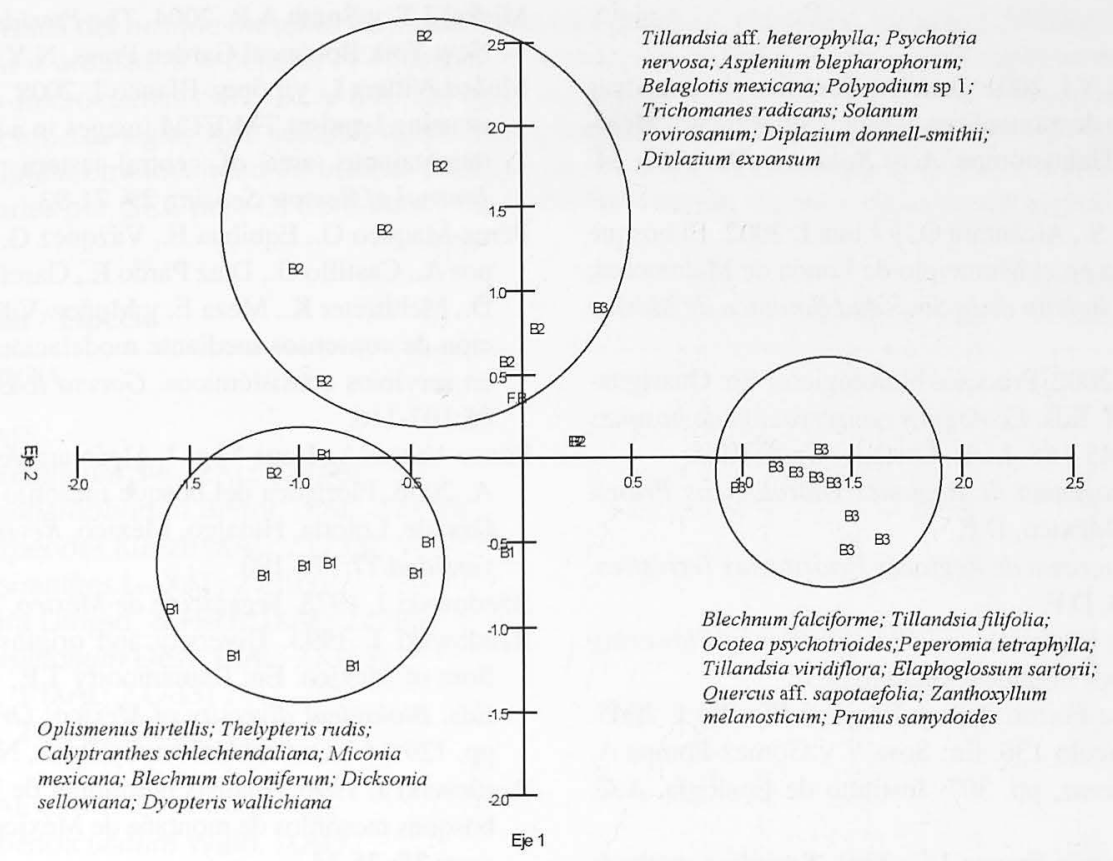

Figura 4. Análisis de componentes principales donde se muestra la separación de las tres microcuencas, y la agrupación de los cuadros de cada una de las microcuencas. Los cuadros de una misma microcuenca se indican con símbolos similares. Las especies al lado de los círculos corresponden con aquellas que caracterizan la agrupación.

con anterioridad (Rzedowski, 1996; Luna et al., 2001); al igual que se ha señalado su variabilidad a nivel regional en el centro de Veracruz (Luna et al., 1988; Williams-Linera, 2007). Sin embargo, hasta donde sabemos, cambios en composición y reemplazo de especies entre sitios ubicados en un mismo fragmento de BMM no habían sido registrados con anterioridad. Aparentemente, el sitio de la Cortadura, por su orografía extremadamente quebrada, limita la existencia y la abundancia de muchas especies en ciertas partes del sitio. Lo anterior significa que, la conservación del remanente de BMM ubicado en La Cortadura, es sumamente prioritario, ya que estos hábitats contienen a las especies características de este tipo de comunidad, así como especies raras (nuevos registros) o únicas (especies nuevas).

\section{Agradecimientos}

Al Municipio de Coatepec y a "Leónides" por permitirnos trabajar en sus predios, y su ayuda en el campo. A M.J. Ordoñez, J. Pale y R. Madrigal por su ayuda en la colecta y preparación de los ejemplares. R. Landgrave elaboró el mapa de ubicación del sitio de estudio. Las acertadas sugerencias y comentarios de dos revisores anónimos y la editora mejoraron el escrito. El estudio fue apoyado por el Consejo Nacional de Ciencia y Tecnología (CONACYT No. 43082) y el Instituto de Ecología, A.C. (INECOL, 902-10/134 GCC; 2011/10-280 GVH; 2011/10-144 JGGF, 2011/10-796 KM, 2011/10-516 MLM).

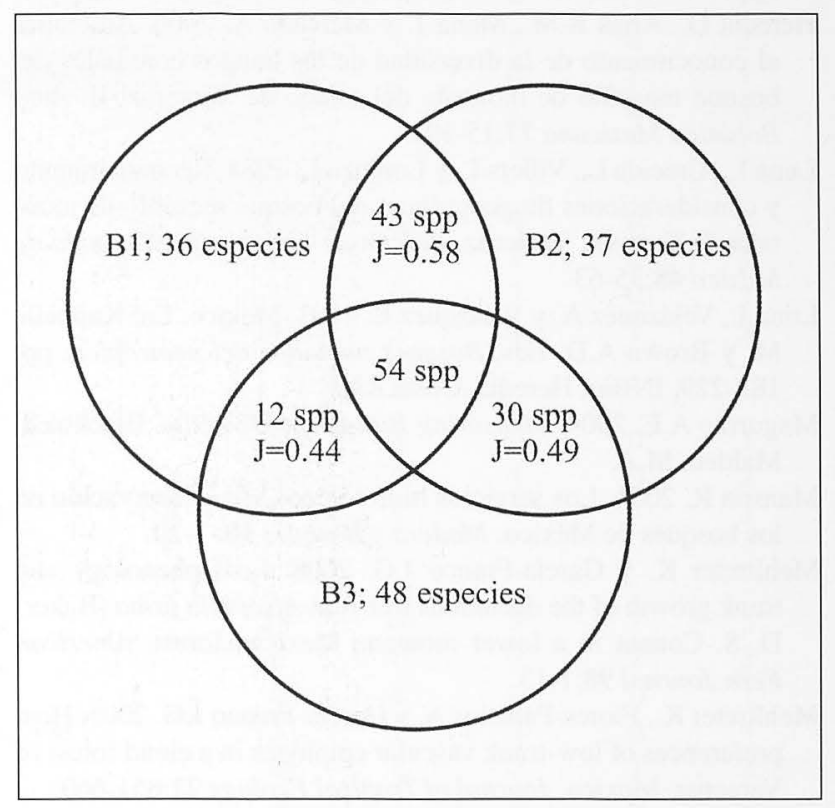

Figura 5. Especies exclusivas, compartidas entre pares de microcuencas y compartidas por todas las microcuencas, señaladas en las áreas en que se traslapan los círculos. B1 = microcuenca $1, \mathrm{~B} 2=$ microcuenca 2, B3 = microcuenca 3. J indica el valor del Índice de Similitud de Jaccard entre pares de microcuencas. 


\section{Literatura Citada}

Alcántara A.O. y Luna V.I. 2001. Análisis florístico de dos áreas con bosque mesófilo de montaña en el estado de Hidalgo, México: Eloxochitlán y Tlahuelompa. Acta Botánica Mexicana 54: 51-87.

Cartujano S., Zamudio S., Alcántara O. y Luna I. 2002. El bosque mesófilo de montaña en el Municipio de Landa de Matamoros, Querétaro, México. Boletín de la Sociedad Botánica de México 70: $13-43$

Cavelier J. y Vargas G. 2002. Procesos hidrológicos. En: Guariguata M.R. y Catan G.H. Eds. Ecología y conservación de bosques neotropicales, pp. 145-165, LUR, Cartago, Costa Rica.

CONABIO. 2000a. Programa de Regiones Hidrológicas Prioritarias. CONABIO, México, D.F.

CONABIO. 2000b. Programa de Regiones Prioritarias Terrestres. CONABIO, México, D.F.

Cronquist A. 1988. The Evolution and Classification of Flowering Plants. The New York Botanical Garden, N.Y.

Espejo-Serna A., López-Ferrari A.R. y Ramírez-Morillo I. 2005. Bromeliaceae. Fascículo 136. En: Sosa V y Gómez-Pompa A. Eds. Flora de Veracruz, pp. 307. Instituto de Ecología, A.C. Xalapa, Ver.

Flores-Palacios A. y García-Franco J.G. 2001. Sampling methods for vascular epiphytes: their effectiveness in recording species richness and frequency. Selbyana 22: 181-191.

Flores-Palacios A. y García-Franco J.G. 2006. The relationship between tree size and epiphyte richness: testing four different hypotheses. Journal of Biogeography 33:323-330.

Heredia G., Arias R.M., Mena J. y Mercado A. 2006. Adiciones al conocimiento de la diversidad de los hongos conidiales del bosque mesófilo de montaña del estado de Veracruz, II. Acta Botánica Mexicana 77:15-30.

Luna I., Almeida L., Villers L. y Lorenzo L. 1988. Reconocimiento y consideraciones fitogeográficas del bosque mesófilo de montaña de Teocelo, Veracruz. Boletín de la Sociedad Botánica de México 48:35-63.

Luna I., Velázquez A. y Velázquez E. 2001. México. En: Kappelle M. y Brown A.D. Eds. Bosques nublados del neotrópico, pp. 183-229, INBio, Heredia, Costa Rica.

Magurran A.E. 2004. Measuring Biological Diversity. Blackwell, Malden, M.A.

Manson R. 2004. Los servicios hidrológicos y la conservación de los bosques de México. Madera y Bosques 10: 3-20.

Mehltreter K. y García-Franco J.G. 2008. Leaf phenology and trunk growth of the deciduous tree fern Alsophila firma (Baker) D. S. Conant in a lower montane Mexican forest. American Fern Journal 98:1-13.

Mehltreter K., Flores-Palacios A. y García-Franco J.G. 2005. Host preferences of low-trunk vascular epiphytes in a cloud forest of Veracruz, Mexico. Journal of Tropical Ecology 21:651-660.
Mickel J.T. y Smith A.R. 2004. The Pteridophytes of Mexico. The New York Botanical Garden Press, N.Y.

Muñoz-Villers L. y López-Blanco J. 2008. Land use/cover changes using Landsat TM/ETM images in a tropical and biodiverse mountainous area of central-eastern Mexico. International Journal of Remote Sensing 29: 71-93.

Pérez-Maqueo O., Equihua E., Vázquez G., Martínez M.L., Campos A., Castillo G., Díaz Pardo E., García-Franco J.G., Geissert D., Mehltreter K., Meza E. y Muñoz-Villers L. 2007. Construcción de consensos mediante modelación mediada con enfoque en servicios ecosistémicos. Gaceta INE Número Especial 8485:107-116.

Ponce-Vargas A., Luna-Vega I, Alcántara-Ayala O. y Ruíz-Jiménez A. 2006. Florística del bosque mesófilo de montaña de Monte Grande, Lolotla, Hidalgo, México. Revista Mexicana de Biodiversidad 77:177-190.

Rzedowski J. 1978. Vegetación de México. Limusa, México, D.F.

Rzedowski J. 1993. Diversity and origins of the phanerogamic flora of Mexico. En: Ramamoorty T.P., Bye R., Lot A. y Fa J. Eds. Biological diversity of Mexico: Origins and distribution, pp. 129-144, Oxford University Press, N.Y.

Rzedowski J. 1996. Análisis preliminar de la flora vascular de los bosques mesófilos de montaña de México. Acta Botánica Mexicana 35: 25-44.

SEmarnat [Secretaría del Medio Ambiente y Recursos Naturales]. 2002. Norma Oficial Mexicana NOM-059-ECOL-2001, Protección ambiental-Especies nativas de México de flora y fauna silvestres-Categorías de riesgo y especificaciones para su inclusión, exclusión o cambio-Lista de especies en riesgo. Diario Oficial de la Federación. 2a Sección, 6 de marzo de 2002.

Sosa V. y Gómez-Pompa A. (comp.). 1994. Lista Florística. Fascículo 82. En: Sosa V. y Gómez-Pompa A. Eds. Flora de Veracruz, pp. 245. Instituto de Ecología, A.C., Xalapa, Ver.

Williams-Linera G. 1993. Bordes de bosque nublado en el Parque Ecológico Clavijero, Xalapa, Veracruz, México. Revista de Biología Tropical 41: 107-117.

Williams-Linera G. 1997. Phenology of deciduous and broadleaved-evergreen tree species in a Mexican tropical lower montane forest. Global Ecology and Biogeography 6: 115-127.

Williams-Linera G. 2002. Tree species richness complementarity, disturbance and fragmentation in a Mexican tropical montane cloud forest. Biodiversity and Conservation 11: 1825-1843.

Williams-Linera G. 2007. El Bosque de niebla del centro de Veracruz: Ecología, historia y destino en tiempos de fragmentación y cambio climático. INECOL-CONABIO, Xalapa, Ver.

Williams-Linera G., Palacios-Rios M. y Hernández-Gómez R. 2005. Fern richness, tree species surrogacy and fragment complementarity in a Mexican tropical montane cloud forest. Biodiversity and Conservation 14: 119-133.

Zar J.H. 1996. Biostatistical Analysis. Prentice Hall, N.J.

Recibido: 8 de enero de 2008

Aceptado: 2 de septiembre de 2008 
Apéndice 1. Lista florística del bosque mesófilo de montaña de La Cortadura, Coatepec, Veracruz, México. Se indica la forma de vida ( $\mathrm{FB}: \mathrm{A}=$ árbol, $\mathrm{Ar}=$ arbusto, $\mathrm{B}=$ bejuco, $\mathrm{H}=$ hierba), el número de cuadros en los que se registraron en el bosque (BMM) y en cada una de las microcuencas (B1, B2 y B3). De igual manera se presenta el número de microcuencas (MC) en que se encontraron las especies. Las siglas XAL señalan que el ejemplar ya se encontraba depositado en el Herbario del Instituto de

Ecología, A.C. para alguna otra localidad de bosque mesófilo de montaña de Veracruz. El número corresponde a la secuencia de ejemplares colectados por GCC para La Cortadura y los ejemplares fueron también depositados en XAL.

\section{FB Grupo / Familia / Especie}

\section{PTERIDOPHYTA}

\section{ASPLENIACEAE}

H Asplenium auriculatum Sw. (XAL; 22248)

$\mathrm{H}$ Asplenium blepharophorum Bertol. (XAL; 21719)

$\mathrm{H}$ Asplenium harpeodes Kunze (XAL; 21733)

H Asplenium monanthes L. (XAL; 21393)

H Asplenium serra Langsd. \& Fisch. (XAL; 22765)

- $\mathrm{H}$ Asplenium sessilifolium Desv. (XAL; 21363)

H Asplenium sp. 1 (XAL; 21735)

H Asplenium sp. 2 (XAL; 21682)

Frecuencia

$\begin{array}{lllll}\text { BMM } & \text { B1 } & \text { B2 } & \text { B3 } & \text { MC }\end{array}$

\section{BLECHNACEAE}

H Blechnum appendiculatum Willd. (XAL)

H Blechnum falciforme (Liebm.) C. Chr. (XAL; 21862)

$\mathrm{H}$ Blechnum fragile (Liebm.) C.V. Morton \& Lellinger (XAL)

$\mathrm{H}$ Blechnum stoloniferum (Mett. ex E. Fourn.) C. Chr. (XAL)

$\mathrm{H}$ Blechnum wardiae Mickel \& Beitel (XAL)

CYATHEACEAE

A Alsophila firma (Baker) D.S. Conant (XAL)

A Cyathea divergens Kunze. var. tuerckheimii (Maxon) Tryon (XAL)

A Cyathea fulva (M. Martens \& Galeotti) Fée (XAL; 22866)

$\begin{array}{ccccc}11 & 6 & 5 & 0 & 2 \\ 3 & 0 & 3 & 0 & 1 \\ 2 & 0 & 1 & 1 & 2 \\ 6 & 0 & 2 & 4 & 2 \\ 5 & 3 & 1 & 1 & 3 \\ 5 & 0 & 4 & 1 & 2 \\ 1 & 0 & 1 & 0 & 1 \\ 1 & 0 & 0 & 1 & 1\end{array}$

\section{DENNSTAEDTIACEAE}

H Dennstaedtia cornuta (Kaulf.) Mett. (XAL)

H Dennstaedtia sp. (XAL)

H Hypolepis blepharochlaena Mickel \& Beitel (XAL)

$\mathrm{H}$ Hypolepis nigrescens Hook. (XAL)

$\begin{array}{lllll}1 & 0 & 0 & 1 & 1 \\ 7 & 1 & 0 & 6 & 2 \\ 4 & 0 & 2 & 2 & 2 \\ 6 & 6 & 0 & 0 & 1 \\ 6 & 2 & 4 & 0 & 2\end{array}$

\section{DICKSONIACEAE}

A Dicksonia sellowiana Hook. (XAL)

A Dicksonia sp. (XAL)

H Lophosoria quadripinnata (J.F. Gmel.) C. Chr. (XAL; 22841)

$\begin{array}{ccccc}7 & 3 & 4 & 0 & 2 \\ 1 & 0 & 0 & 1 & 1 \\ 15 & 9 & 2 & 4 & 3\end{array}$

\section{DRYOPTERIDACEAE}

H Arachniodes denticulata (Sw.) Ching (XAL; 21362)

$\mathrm{H}$ Ctenitis cf. hemsleyana (Baker ex Hemsl.) Copel. (XAL)

H Dryopteris wallichiana (Spreng.) Hyl. (XAL)

H Megalastrum pulverulentum (Poir.) A.R. Sm. \& R.C. Moran (XAL)

H Polystichum fournieri A.R. Sm. (XAL; 21368)

H Polystichum sp. 1 (XAL; 21368)

H Polystichum sp. 2 (XAL; 21515)

$\begin{array}{ccccc}10 & 6 & 4 & 0 & 2 \\ 1 & 0 & 1 & 0 & 1 \\ 1 & 0 & 1 & 0 & 1 \\ 6 & 3 & 2 & 1 & 3\end{array}$

\section{GRAMMITIDACEAE}

H Cochlidium linearifolium (Desv.) Maxon ex C. Chr. (XAL)

H Lellingeria prionodes (Mickel \& Beitel) A.R. Sm. \& R.C. Moran (XAL)

H Melpomene leptostoma (Fée) A.R. Sm. \& R.C. Moran (XAL)

H Terpsichore cultrata (Bory ex Willd.) A.R. Sm. (XAL; 22294)

$\begin{array}{lllll}8 & 8 & 0 & 0 & 1 \\ 3 & 2 & 1 & 0 & 2 \\ 9 & 1 & 1 & 7 & 3\end{array}$

$\begin{array}{ccccc}24 & 10 & 7 & 7 & 3 \\ 5 & 0 & 3 & 2 & 2 \\ 6 & 6 & 0 & 0 & 1 \\ 3 & 0 & 3 & 0 & 1 \\ 9 & 6 & 2 & 1 & 3 \\ 4 & 1 & 3 & 0 & 2 \\ 9 & 3 & 6 & 0 & 2\end{array}$




\section{GLEICHNIACEAE}

H Sticherus palmatus (W. Schaffn. ex E. Foum.) Copel. (XAL; 22883)

\section{HYMENOPHYLLACEAE}

H Hymenophyllum polyanthos (Sw.) Sw. (XAL)

H Hymenophyllum sp. (XAL; 21364)

$\mathrm{H}$ Hymenophyllum tegularis (Desv.) Proctor \& Lourteig (XAL)

$\mathrm{H}$ Trichomanes capillaceum L. (XAL)

H Trichomanes radicans Sw. (XAL)

H Trichomanes reptans Sw. (XAL; 21367)

H Trichomanes sp. (XAL; 21268)

\section{LOMARIOPSIDACEAE}

H Elaphoglossum erinaceum (Fée) T. Moore (XAL)

H Elaphoglossum lonchophyllum (Fée) T. Moore (XAL)

H Elaphoglossum peltatum (Sw.) Urb. (XAL)

H Elaphoglossum sartorii (Liebm.) Mickel (XAL; 22867)

H Elaphoglossum sp. 2 (XAL)

H Elaphoglossum sp. 4 (XAL)

\section{LYCOPODIACEAE}

H Huperzia sp. (XAL)

\section{MARATTIACEAE}

H Marattia laxa Kunze (XAL)

\section{POLYPODIACEAE}

H Campyloneurum angustifolium (Sw.) Fée (XAL)

H Phlebodium areolatum (Humb. \& Bonpl. ex Willd.) J. Sm. (XAL)

$\mathrm{H}$ Pleopeltis crassinervata (Fée) T. Moore (XAL)

H Pleopeltis mexicana (Fee) Mickel \& Beitel (XAL)

$\mathrm{H}$ Pleopeltis sp. (XAL)

H Polypodium falcaria Kunze (XAL; 22838)

$\mathrm{H}$ Polypodium longepinnulatum E. Fourn. (XAL; 22863)

H Polypodium plebeium Schltdl. \& Cham. (XAL; 21392)

$\mathrm{H}$ Polypodium plesiosorum Kunze (XAL)

$\mathrm{H}$ Polypodium polypodioides (L.) Watt (XAL)

H Polypodium puberulum Schldl. \& Cham. (XAL)

H Polypodium sp. (XAL; 21516)

\section{PSILOTACEAE}

H Psilotum complanatum Swartz. (XAL; 22764)

PTERIDACEAE

H Adiantum andicola Liebm. (XAL; 22877)

$\mathrm{H}$ Pteris muricata Hook. (XAL)

H Pteris orizabae M. Martens \& Galeotti (XAL)

$\mathrm{H}$ Pteris sp. (XAL)

\section{SELAGINELLACEAE}

H Selaginella sp. 2 (XAL; 22539)

H Selaginella stellata Spring. (XAL; 21679)

\section{THELYPTERIDACEAE}

H Thelypteris rudis (Kunze) Proctor (XAL; 22067)

H Thelypteris sp. (subgen. Amauropelta) (XAL; 22189)

$\begin{array}{ccccc}10 & 8 & 2 & 0 & 2 \\ 1 & 0 & 1 & 0 & 1\end{array}$


$\mathrm{H}$ Diplazium expansum Willd. (XAL)

H Diplazium franconis Liebm. (XAL)

H Diplazium ternatum Liebm (XAL; 21491)

$\begin{array}{ccccc}2 & 0 & 2 & 0 & 1 \\ 2 & 0 & 2 & 0 & 1 \\ 12 & 5 & 7 & 0 & 2 \\ 11 & 5 & 6 & 0 & 2 \\ & & & & \\ 10 & 3 & 5 & 2 & 3 \\ 11 & 3 & 3 & 5 & 3\end{array}$

H Scoliosorus ensiformis (Hook.) T. Moore (XAL)

H Vittaria graminifolia Kaulf. (XAL; 22913)

\section{MONOCOTILEDONEAS}

ARACEAE

H Anthurium andicola Liebm. EM. (XAL; 22871)

$\mathrm{H}$ Anthurium scandens (Aubl.) Engl. (XAL; 21532)

$\mathrm{H}$ Anthurium sp. (cortadura) (XAL)

H Philodendron advena Schott (XAL)

H Philodendron sp. (Cortadura) (XAL)

$\begin{array}{ccccc}1 & 0 & 0 & 1 & 1 \\ 15 & 3 & 5 & 7 & 3 \\ 4 & 2 & 1 & 1 & 3 \\ 7 & 5 & 2 & 0 & 2 \\ 2 & 0 & 0 & 2 & 1\end{array}$

\section{ARECACEAE}

H Chamaedorea klotzschiana H. Wendland. (XAL; 22738)

H Chamaedorea sp. 1 (XAL; 21488)

H Chamaedorea sp. 2 (XAL; 22749)

$\begin{array}{ccccc}12 & 0 & 7 & 5 & 2 \\ 2 & 2 & 0 & 0 & 1 \\ 3 & 0 & 0 & 3 & 1\end{array}$

\section{BROMELIACEAE}

H Catopsis sessiliflora (Ruiz \& Pav.) Mez (XAL)

H Catopsis sp. 1 (XAL)

$\mathrm{H}$ Catopsis spp. (no reproductivas) (XAL)

H Greigia vanhyningii L.B. Sm. (XAL; 21551)

H Racinaea ghiesbreghtii (Baker) M.A. Spencer \& L.B. Sm. (XAL)

$\mathrm{H}$ Tillandsia aff. heterophylla E. Morren (XAL)

H Tillandsia butzii Mez (XAL; 21517)

$\mathrm{H}$ Tillandsia fasciculata Sw. (XAL)

$\mathrm{H} \quad$ Tillandsia filifolia Schltdl. \& Cham. (XAL)

H Tillandsia gymnobotrya Baker (XAL; 21513)

$\mathrm{H}$ Tillandsia heterophylla E. Morren (XAL)

H Tillandsia imperialis E. Morren ex Roezl (XAL; 21779)

H Tillandsia juncea (Ruiz \& Pav.) Poir. (XAL; 22712)

$\mathrm{H}$ Tillandsia kirchhoffiana Wittm. (XAL)

H Tillandsia punctulata Schltdl. \& Cham. (XAL)

$\mathrm{H}$ Tillandsia spp. (no reproductivas) (XAL)

$\mathrm{H}$ Tillandsia viridiflora (Beer) Baker (XAL)

H Werauhia gladioliflora (H. Wendl) J.R. Grant. (XAL)

COMMELINACEAE

H Gibasis pellucida (M. Martens \& Galeotti) D.R. Hunt (XAL; 22047)

H Tradescantia zanonia (L.) Sw. (XAL; 21357)

CONVALLARIACEAE

H Maianthemum paniculatum (M. Martens \& Galeotti) LaFrankie (XAL; 21773) 9

\section{CYPERACEAE}

H Carex donnell-smithii L.H. Bailey (XAL; 21768)

H Rhynchospora aristata Boeck. (XAL; 21770)

H Rhynchospora macrochaeta Steud. ex Boeck. (XAL; 22270)

H Uncinia hamata (Sw.) Urb. (XAL; 21519) 
ORCHIDACEAE

H Beloglottis mexicana Garay \& Hamer (XAL; 21370)

H Coelia sp. (XAL; 22876)

H Dichaea neglecta Schltr. (XAL; 21542)

H Elleanthus cynarocephalus (Rchb. f.) Rchb. f. (XAL; 22875)

H Isochilus unilaterale Robins. (XAL; 22889)

H Lepanthes attenuata Salazar, Soto Arenas \& O. Suárez (XAL; 22892)

H Maxillaria meleagris Lindl. (XAL;22832)

H Orquídea sp. (XAL; 21778)

$\mathrm{H} \quad$ Pleurothallis sp. (XAL)

H Stelis pachyglossa (Lindl.) Pridgeon \& M. W. Chase (XAL; 21531)

\section{POACEAE}

H Ichnanthus nemorosus (Sw.) Döll (XAL; 21519)

H Ichnanthus pallens (Sw.) Munro ex Benth. (XAL; 21369)

H Oplismenus hirtellus (L.) P. Beauv. (XAL; 21776)

H Panicum sp. (XAL; 22858)

\section{PODOCARPACEAE}

A Podocarpus matudae Lundell (XAL; 22884)

\section{SMILACACEAE}

B Smilax jalapensis Schltdl. (XAL; 22459)

B Smilax mollis Humb. \& Bonpl. ex Willd. (XAL; 21775)

B Smilax sp. (XAL; 22132)

\section{DICOTILEDONEAS}

\section{ACANTHACEAE}

Ar Stenostephanus haematodes (Schltdl.) T.F. Daniel (XAL; 22252)

$\begin{array}{ccccc}3 & 0 & 2 & 1 & 2 \\ 4 & 0 & 4 & 0 & 1 \\ 12 & 9 & 3 & 0 & 2 \\ 2 & 0 & 0 & 2 & 1\end{array}$

\section{ACTINIDIACEAE}

A Saurauia yasicae Loes. (XAL; 22761)

2

2

$$
0
$$

3

$$
\begin{aligned}
& 3 \\
& 0 \\
& 0
\end{aligned}
$$$$
\begin{aligned}
& 0 \\
& 0
\end{aligned}
$$$$
1
$$$$
13
$$$$
\begin{array}{ll}
1 & 1
\end{array}
$$$$
53
$$$$
11
$$$$
21
$$$$
1
$$$$
1
$$$$
\begin{array}{ll}
0 & 2
\end{array}
$$$$
\begin{aligned}
& 1 \\
& 0
\end{aligned}
$$$$
1
$$$$
\text { 0 }
$$

1


BMM

22

8

Frecuencia

CACTACEAE

H Nopalxochia phyllanthoides (DC.) Britton \& Rose (XAL; 21481)

CAMPANULACEAE

Ar Centropogon grandidentatus (Schlecht.) Zahlbr. (XAL; 22290)

CAPRIFOLIACEAE

A Sambucus nigra subsp. canadensis L. Bolli (XAL; 21518)

CELASTRACEAE

B Celastrus vulcanicola Donn. Sm. (XAL; 21375)

A Perrottetia ovata Hemsley (XAL;21386)

A Quetzalia stipitata (Lundell) Lundell (XAL; 22281)

\section{CLETHRACEAE}

A Clethra mexicana DC. (XAL; 22263)

A Clethra sp. (XAL)

\section{CHLORANTHACEAE}

A Hedyosmum mexicanum Cordem. (XAL; 21399)

\section{COCHLOSPERMACEAE}

A Cochlospermum vitifolium (Willd.) Spreng. (XAL)

\section{CRASSULACEAE}

$\mathrm{H}$ Echeveria sp. (XAL)

\section{CUCURBITACEAE}

B Cyclanthera langaei Cogn. (XAL; 22247)

B Hanburia mexicana Seem. (XAL; 21378)

\section{CUNONIACEAE}

A Weinmannia pinnata L. (XAL)

\section{ERICACEAE}

A Gynanthes sp. (XAL; 22864)

B Lyania sp. (XAL; 22881)

B Macleania sp. (XAL; 22882)

A Vaccinium leucanthum Cham. \& Schldl. (XAL; 21355)

\section{EUPHORBIACEAE}

H Acalypha alopecuroidea Jacq. (XAL; 21658

A Alchornea latifolia Sw. (XAL; 21523)

Ar Bernardia interrupta (Schltdl.) Müll. Arg. (XAL; 21476)

\section{FABACEAE}

A Cojoba arborea (L.) Britton \& Rose (XAL; 21404)

A Inga schiedeana Steud. (XAL; 21411; 21507)

\section{FAGACEAE}

A Quercus aff. sapotaefolia L. (XAL; 21547)

A Quercus corrugata Hook. (XAL; 21784)

A Quercus laurina Humb. \& Bonpl. (XAL)

A Quercus sp. (XAL; 22130)

A Quercus xalapensis Humb. \& Bonpl. (XAL) 
GESNERIACEAE

Ar Moussonia deppeana (Schlecht. \& Cham.) Hanst. (XAL; 21408)

\section{HAMAMELIDACEAE}

A Liquidambar styraciflua L. (XAL)

\section{LAMIACEAE}

H Salvia tiliifolia var. albiflora (M. Martens \& Galeotti)

L.O. Williams (XAL; 22249)

\section{LAURACEAE}

A Cinnamomum effusum (Meisn.) Kosterm (XAL; 22275)

A Ocotea disjuncta Lorea-Hern. (XAL; 21545)

A Ocotea effusa (Meisn.) Hemsl. (XAL; 21385)

A Ocotea psychotrioides Kunth (XAL; 22861)

A Persea americana Mill. (XAL; 21553)

MAGNOLIACEAE

A Magnolia schiedeana Schltdl. (XAL; 22857)

\section{MARCGRAVIACEAE}

B Marcgravia stonei Utley (XAL)

\section{MELASTOMATACEAE}

A Conostegia arborea (Schltdl.) Steud (XAL; 22743)

A Miconia chrysoneura Triana (XAL; 22757)

A Miconia glaberrima (Schltdl.) Naudin (XAL; 22887)

A Miconia mexicana (Bonpl.) Naudin (XAL; 22075)

A Miconia oligotricha (DC.) Naudin (XAL; 21771)

MELIACEAE

A Guarea grandifolia DC. (XAL; 22741)

A Trichilia minutiflora Standl. (XAL; 21793)

$\begin{array}{ccccc}9 & 5 & 4 & 0 & 2 \\ 6 & 0 & 2 & 4 & 2 \\ 4 & 2 & 1 & 1 & 3 \\ 6 & 0 & 0 & 6 & 1 \\ 4 & 0 & 1 & 3 & 2 \\ & & & & \\ 3 & 0 & 0 & 3 & 1 \\ & & & & \\ 4 & 0 & 0 & 4 & 1 \\ & & & & \\ & & & & 1 \\ 1 & 0 & 0 & 1 & 3 \\ 18 & 10 & 7 & 1 & 3 \\ 16 & 1 & 6 & 9 & 3 \\ 7 & 6 & 1 & 0 & 2 \\ 3 & 3 & 0 & 0 & 1\end{array}$

\section{MORACEAE}

A Trophis mexicana (Liebm.) Bureau (XAL; 22735)

$\begin{array}{lllll}4 & 0 & 2 & 2 & 2\end{array}$

MYRSINACEAE

A Parathesis melanosticta (Schltdl.) Hemsl. (XAL; 22932)

A Myrsine coriacea (Sw.) R. Br. Ex Roem. \& Schult. (XAL)

$\begin{array}{lllll}25 & 10 & 10 & 5 & 3\end{array}$

MYRTACEAE

Ar Calyptranthes schlechtendaliana O. Berg. (XAL; 21786)

$\begin{array}{lllll}13 & 8 & 5 & 0 & 2\end{array}$

ONAGRACEAE

Ar Fuchsia microphylla Kunth (XAL; 22259)

PASSIFLORACEAE

B Passiflora sexflora A.L. Juss. (XAL; 22777)

A Phyllonoma laticuspis (Turcz.) Engl. (XAL; 22718)

$\begin{array}{llll}5 & 9 & 8 & 3 \\ 7 & 3 & 1 & 3\end{array}$


H Peperomia deppeana Schldl. \& Cham. (XAL; 22288)

B1

B2

B3

MC

Ar Peperomia donaguiana DC. (XAL; 22730)

H Peperomia glabella (Sw.) A. Dietr. (XAL)

H Peperomia obtusifolia (L.) A. Dietr. (XAL; 22463)

H Peperomia sp. (XAL; 21774)

H Peperomia tetraphylla (G. Forst.) Hook. \& Arn. (XAL; 22766)

Ar Piper amalago L. (XAL; 22744)

Ar Piper hispidum Sw. (XAL; 22190)

Ar Piper nudum C. DC. (XAL; 21486)

Ar Piper sp. (XAL; 22462)

H Piper xanthostachyum C. DC. (XAL; 22261)

$\begin{array}{ccccc}13 & 0 & 6 & 7 & 2 \\ 12 & 0 & 4 & 8 & 2 \\ 10 & 3 & 5 & 2 & 3 \\ 4 & 1 & 3 & 0 & 2 \\ 4 & 0 & 2 & 2 & 2 \\ 6 & 0 & 0 & 6 & 1 \\ 3 & 1 & 1 & 1 & 3 \\ 2 & 0 & 1 & 1 & 2 \\ 5 & 2 & 3 & 0 & 2 \\ 2 & 0 & 2 & 0 & 1 \\ 17 & 10 & 7 & 0 & 2\end{array}$

\section{RHAMNACEAE}

A Rhamnus longistyla C.B. Wolf (XAL; 22709)

A Rhamnus sp. (XAL; 22885)

\section{ROSACEAE}

Ar Prunus samydoides Schltdl. (XAL; 22676)

Ar Rubus adenotrichus Schltdl. (XAL; 21497)

Ar Rubus coriifolius Liebm. (XAL; 21549)

Ar Rubus sp.

\section{RUBIACEAE}

A Arachnothryx bourgaei (Standl.) Borhidi (XAL; 22739)

A Arachnothryx capitellata (Hemsl.) Borhidi (XAL; 22454)

H Crusea coccinea DC. (XAL; 22282)

Ar Deppea grandiflora Schltdl. (XAL; 22660)

H Didymaea alsinoides (Schltdl. \&Cham.) Standl. (XAL; 21791)

Ar Hoffmannia excelsa (Kunth) K. Schum. (XAL; 21484)

Ar Hoffmannia psychotriifolia (Benth.) Griseb. (XAL; 22671)

A Psychotria galeottiana (M. Martens) C.M. Taylor \& Lorence (XAL)

Ar Psychotria nervosa Sw. (XAL; 22485)

Ar Psychotria sp. (XAL; 21506)

H Spermacoce tenuior L. (XAL; 22083)

\section{RUTACEAE}

$\begin{array}{ccccc}10 & 0 & 4 & 6 & 2 \\ 6 & 1 & 3 & 2 & 3 \\ 1 & 1 & 0 & 0 & 1 \\ 10 & 4 & 5 & 1 & 3 \\ 3 & 3 & 0 & 0 & 1 \\ 27 & 10 & 10 & 7 & 3 \\ 1 & 0 & 1 & 0 & 1 \\ 2 & 2 & 0 & 0 & 1 \\ 7 & 1 & 6 & 0 & 2 \\ 17 & 5 & 2 & 10 & 3 \\ 2 & 1 & 1 & 0 & 2\end{array}$

A Citrus limettioides Tanaka (XAL; 21376)

A Citrus sp. (XAL)

A Zanthoxylum juniperinum Poepp. (XAL; 21768)

A Zanthoxylum melanostictum Schltdl. \& Cham (XAL; 21667)

\section{SABIACEAE}

A Meliosma alba (Schltdl.) Walp. (XAL)

SAPINDACEAE

B Serjania sp. (XAL; 21383)

A Sideroxylon capiri (A. DC.) Pittier (XAL)

A Sideroxylon sp. (XAL)

SIMAROUBACEAE

Ar Picramnia antidesma Sw. (XAL; 22860)

\section{SOLANACEAE}

Ar Cestrum elegans (Brongn.) Schltdl. (XAL; 22724, 22292)

Ar Cestrum sp. (XAL)

Ar Lycianthes geminiflora (Martens \& Galeotti) Bitter. (XAL; 22271)

0
1

0

21
1

2
3
1
3
1
3
1
1
2
3
2


Frecuencia

Ar Solanum nigricans M. Martens \& Galeotti (XAL; 21381; 22246)

Ar Solanum nudum Dunal (XAL; 22260)

Ar Solanum pubigerum Dunal (XAL; 21797)

Ar Solanum rovirosanum Donn. Sm. (XAL; 21522)

Ar Solanum sp. (XAL; 22246)

Ar Witheringia solanacea L’Her. (XAL; 21495)

STAPHYLEACEAE

A Turpinia occidentalis (Swartz) G. Don. (XAL; 22935)

STYRACACEAE

A Styrax glabrescens Benth. (XAL; 21524)

\section{SYMPLOCACEAE}

A Symplocos coccinea Humb. \& Bonpl. (XAL; 21502)

\section{THEACEAE}

A Ternstroemia sylvatica Schltdl. \& Cham. (XAL; 22897)

THYMELAEACEAE

A Daphnopsis americana (Mill.) J.R. Johnst. (XAL; 21526)

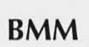

\section{B2}

B3 $\mathrm{MC}$

6

\section{5}

11

$1 \quad 1$

$5 \quad 0$

2

32

1

0

0

2

0

$0 \quad 1$

5

2

$\begin{array}{ll}0 & 1\end{array}$

$0 \quad 1$

0 2

0

2

$\begin{array}{ll}0 & 1\end{array}$

\section{URTICACEAE}

H Pilea pubescens Liebm. (XAL; 21492)

H Pilea riparia Donn. Sm. (XAL; 22256)

Ar Urera caracasana (Jacq.) Gaudich. ex Griseb. (XAL; 21525)

Ar Urera sp. (XAL; 21380)

\section{VERBENACEAE}

B Aegiphila deppeana Steud. (XAL; 22480)

\section{VIBURNACEAE}

Ar Viburnum microcarpum Schltdl. \& Cham. (XAL; 21782)

\section{VISCACEAE}

Ar Phoradendron sp. 2 (XAL)

\section{VITACEAE}

B Vitis bourgaeana Planch. (XAL; 21400) 